\title{
Kinetics of IgA and eosinophils following a low-dose, predominantly Haemonchus contortus infection of Boer goats
}

\begin{abstract}
Aims: Most breeds of goat are more susceptible to nematode infection than sheep, and this appears to be a consequence of less effective immune responses. Several papers have considered the effectiveness of eosinophils and immunoglobulin $\mathrm{A}(\operatorname{Ig} \mathrm{A})$ in goats but differences in the induction of responses have not been studied in the same detail. The aim of this study was to look at the induction of eosinophil and $\operatorname{IgA}$ responses in Boer goats reared indoors under intensive conditions. Method and results: The goats were experimentally infected with a low dose of 2400 Haemonchus contortus, Trichostrongylus spp. and Oesophagostomum spp. at a 6:1:1 ratio. Faecal egg counts (FEC), packed cell volume (PCV), IgA activity against third-stage larvae and peripheral eosinophilia were measured twice a week for eight weeks. The infection generated an IgA response but did not significantly increase peripheral eosinophilia in the 25 infected kids compared with the 4 control animals. FEC was not associated with IgA activity or eosinophilia. Conclusion: A detailed analysis of $\operatorname{Ig} \mathrm{A}$ and eosinophil responses to deliberate nematode infection in Boer goats showed that there was an increase in nematode-specific IgA activity but no detectable eosinophil response. In addition, there was no association between increased $\operatorname{IgA}$ activity or eosinophilia with egg counts and worm burdens. These suggest that IgA and eosinophils do not act to control nematode infection in goats.
\end{abstract}

